\title{
PEINTURE ET GÉOGRAPHIE
}

\author{
JEAN-LUC PIVETEAU
}

Les peintres - ceux du moins qui peignent des paysages - et les géographes, ont au fond un objet commun d'intérêt: le visage de la Terre; ce visage que définissent les interférences subtiles de plusieurs enveloppes imbriquées: lithosphère, atmosphère, biosphère et noosphère.

Je ne sais si les peintres songent quelquefois aux géographes. A coup sûr, en tant que géographe, je pense souvent aux peintres. Leurs tableaux me paraissent suscept:bles d'apporter quelque chose à notre vision scientifique du globe, et de contribuer, dans une certaine mesure, à nous révéler des aspects de la réalité que nous n'avons pas su voir. Est-ce une impression dangereuse?

Mises à part quelques pages aussi pertinentes que brèves de M. Georges Chabot sur les erreurs géomorphologiques commises par les paysagistes ${ }^{1}$, et d'André Allix sur les ambiguités du dessin², il n'existe semble-t-il, aucune référence à la possibilité d'un tel rapprochement dans la bibliographie géographique de langue française. Et s'il y a quelque chose de stimulant pour l'esprit à essayer un sentier nouveau, le silence quasi général intimide. Nombreux, certes, sont les critiques d'art comme les philosophes, qui parlent des rapports entre la peinture et le réel. Mais leur point de vue n'est et ne peut être celui du géographe. Or, c'est précisément sous l'angle de notre discipline que je voudrais aborder, ici, ce problème.

\section{Une cause apparemment difficile à défendre}

Qui refuserait de reconnaître qu'à première vue il y a un paradoxe à prétendre que le tableau de peintre puisse tenir lieu de document valable pour le géographe?

Sans doute certaines toiles nous confondent-elles par leur fidélité au paysage - présent ou passé - qu'elles représentent. Vermeer dans sa célèbre «Vue de Delft», Courbet dans «La roche de 10 heures» ont littéralement photographié ce qu'ils ont vu. Un livre comme celui de L. Reidemeister ${ }^{3}$ rassemble une impressionnante collection d'œuvres récentes, en regard desquelles il est possible de faire correspondre un cliché d'une similitude frappante. Et parmi les peintures qui ne montrent pas autant de ressemblance avec un lieu défini, plusieurs demeurent d'étonnants témoignages. Que l'on songe, par exemple, à ce panorama de Dürer, exposé au musée de Berlin, ou à cette «Entrée de village» de Corot qui figure au Louvre. Pourrait-on accuser les auteurs d'avoir triché en esprit? Ne nous offrent-ils pas là des «types» bien caractérisés d'habitat rural ?

Il n'en reste pas moins vrai que, dans la majorité des cas, la recherche d'une correspondance exacte entre l'image de l'artiste et la réalité est illusoire. Le concordisme reste finalement limité dans le nombre de ses applications, et toujours risqué, même là où il apparaît plausible.

Les méthodes, les motifs, et finalement le langage du peintre sont en effet spécifiques.

Les paysagistes travaillent volontiers de mémoire. Sur le vif ils prennent des esquisses. Mais c'est en atelier, bien souvent, qu'ils font véritablement le tableau : le service d'ordre dont Degas réclamait la constitution pour «canarder» tous ceux que l'on surprendrait, dans la nature, un pinceau à la main, n'eût fait selon toute vraisemblance qu'un nombre modéré de victimes. Un tel processus de création fondé sur le souvenir, conduit tout

1 G. Chabot: «Peinture et géographie». Information géographique, 1949, p. 78.

2 A. Allix: «Images et morphologie». Mélanges géographiques offerts à Ph. Arbos, ClermontFerrand, 1953, pp. 7-14.

3 Reidemeister, L.: «Auf den Spuren der Maler der Ile de France», Berlin 1963.

4 «Die Drahtziehmühle». 
naturellement le peintre à en user très librement avec la composition. Il choisit, élaguant tel trait, ajoutant tel autre, rapprochant ou même télescopant plusieurs éléments qui, dans la réalité temporelle ou spatiale, se trouvaient éloignés les uns des autres: il recrée.

Cette désinvolture tient à ce que chez lui les mobiles ne sont pas «scientifiques»au sens courant du mot. Le peintre ne cherche pas à expliquer ce qu'il voit - ni même peutêtre à décrire, au premier chef, l'apparence des choses. Ce qui ne signifie pas qu'il se désintéresse de l'exactitude. Mais il poursuit une vérité différente, d'ordre plastique surtout, ou d'ordre éthique. Ainsi, très souvent, la représentation figurée vise-t-elle seulement à polariser l'attention du spectateur sur une certaine qualité irréelle de la lumière et l'état d'âme qu'elle engendre - par exemple dans une composition enneigée d'impressionniste; sur le tumulte baroque des cumulonimbus face à la lourdeur étirée du relief terrestre ( «Le coup de soleil» de Ruysdael) ; sur l'odeur pénétrante de touffes buissonnantes dans l'air chaud qui vibre («Les Peyroulets» de Van Gogh).

S'il s'y ajoute ou s'il s'y substitue la quête d'un message ou d'une quintessence, le goût du symbolisme ou de la stylisation, tout essai de relation avec ce qui se voit devient difficile. L'étonnant panorama de Villon «Entre Toulouse et Albi» - où peut-être d'aucuns morphologues retrouveraient une pénéplaine éogène -, le «Paysage grec» de Lurçat, s'éloignent à grandes enjambées du document figuratif utilisable. Et que dire à la limite de ces compositions, pourtant scrupuleuses quant aux formes de détail, qui représentent des contrées mythiques? Où localiser sur un planisphère les hautes frondaisons d'un Poussin ou d'un Watteau? Les mondes imaginaires, à coup sûr, ne peuvent plus servir d'instrument: ils seront le repos du géographe.

La subjectivité du langage pictural nous engage dans une voie plus contestable encore. On accordera volontiers que le peintre fait æuvre collective dans la mesure où, placé devant un paysage, il s'efforce soit de traduire ce que d'autres ressentent, mais sont dans l'incapacité de formuler, soit de révéler ce que, lui, voit, pour que ceux qui regarderont sa toile l'éprouvent à leur tour. La peinture n'en devient pas pour autant moyen de communication. Elle reste avant tout instrument d'expression lié à chaque créateur, et variable, donc, autant qu'ils sont différents. Elle ne possède pas de référence indépendante de ceux qui la pratiquent. Elle oblige le spectateur à réétalonner sans cesse ses critères de jugement.

Ce manque fondamental d'objectivité se vérifie aisément.

D'une même réalité, les peintres donnent chacun une interprétation particulière. Tributaires de leur «race», de leur culture, parties prenantes du milieu socio-économique qui les a formés, témoins de leur temps, ils possèdent aussi une personnalité propre. René Huyghe, comparant «La pêche miraculeuse» de Conrad Witz avec le «Lac de Thoune» de Turner, constate que le Suisse médiéval minimise le relief alors que le romantique anglais le dramatise. Les rues d'Auvers se métamorphosent de Van Gogh à Cézanne. Du village d'Ile de France, le nordique enfiévré a rendu quasi charnellement l'individualité et l'intimité des maisons. Plus attentif au petit troupeau des toits aiguisés, le méditerranéen consciencieux en a, lui, saisi l'impression de juste mesure.

A l'inverse, sous le pinceau d'un même peintre, des régions éloignées prennent des tonalités très voisines. Que l'on juxtapose par exemple à tel «Souvenir d'Italie» de Corot cette «Levée de filet» du même auteur, inspirée par les étangs de Ville d'Avray: une buée commune les enveloppe; la végétation se prolonge d'une toile à l'autre, et, n'était le profil des maisons comme une certaine coloration de l'air, on ferait confluer l'Arno et la Seine dans une Sologne méridionale dont Corot seul connaîtrait l'accès. Dunoyer de Segonzac imprègne de la même sévérité hivernale son «Golfe de Saint Tropez» et sa vue de «La vallée de la Bièvre à Jouy en Josas». Il serait facile de multiplier les exemples.

Faut-il en conclure, dès à présent, que le procès est entendu, que géographie et peinture n'ont que bien peu à voir l'une avec l'autre; et que, de même que l'oratoire et le 
laboratoire selon Pasteur, le goût de la cimaise et l'analyse du paysage chez le géographe ne nourriraient que l'équivoque à se frôler?

\section{Pour une double justification}

Sans rien nier de ce qui précède, il ne me paraît cependant pas douteux que notre connaissance de la Terre n'ait à tirer profit d'une fréquentation des peintres.

A bien de leurs toiles on pourrait être tenté, de prime abord, d'accorder un mérite: celui de constituer un document original, intermédiaire entre la description et la photographie. Comme celle-ci le tableau permet, pour un paysage que l'on chercherait à connaître, une approche immédiate et synoptique. Comme celle-là, il offre l'avantage d'une initiation à la fois sélective et synthétique. Ce double caractère, des géographes - des morphologues surtout - le reconnaissent qui, s'ils disposent d'aptitude au dessin, n'hésitent pas à ajouter à leur texte déjà illustré de clichés, des croquis personnels.

Une comparaison entre les trois moyens d'expression ne manque pas d'être suggestive. Rapprochons, par exemple, de la «Tolède» du Gréco, d'une part la description de la ville telle que nous la donne Barrès dans un style exemplaire, et, d'autre part, une photo de la cité castillane prise sous un angle de vue semblable à celui, commun, du peintre et de l'écrivain. Avec toute la prudence et toute la réserve qu'impose une confrontation de documents d'époques bien différentes, il reste possible de saisir la spécificité du témoignage pictural. Le cliché, d'une fidélité inerte au réel, livre tout. A l'observateur d'en organiser l'inventaire. Cette mise en ordre, l'évocation par le langage la fournira, elle, mais pièce à pièce: qu'on relise les pages de Barrès. Regardons enfin le tableau. Le peintre nous donne d'emblée, ce que la description écrite ne peut que dérouler dans le temps. Et mieux que la photo, sa toile aura travaillé pour nous, négligeant le détail, exaltant les contrastes. Il n'est que de voir comment Barrès s'inspire du Gréco, pour comprendre à quel point la vision du peintre peut faciliter, parfois, une prise de conscience.

L'usage comme tel de la peinture requiert de toute évidence des précautions, car les critères de sélection de l'artiste ne sont pas nécessairement raisonnés. Ne visant ni à reconstituer une genèse, ni à imposer une démonstration, ils restent beaucoup plus instinctifs et visuels. Et si cet affranchissement à l'égard d'un quelconque esprit de système, tient lieu, à sa manière, de caution d'indépendance dans l'observation, il risque de faire passer le spectateur à côté d'aspects importants de la réalité physique ou humaine.

C'est donc ailleurs qu'il faut trouver des justifications de notre recours aux peintres. J'en distinguerais, pour ma part, deux essentielles. Le tableau en premier lieu enrichit notre vision du monde en nous incitant à réhabiliter une certaine subjectivité - et en nous révélant les limites de ce que nous prétendons être de l'objectivité dans nos analyses de paysages.

A force de vouloir évacuer, par rigueur scientifique, tout caractère personnel dans nos descriptions, n'en venons-nous pas à donner une image appauvrie de la réalité? Même sans aller jusqu'à dire que celle-ci est relative à chacun d'entre nous, ne peut-on pas estimer que la vision très, trop subjective souvent du peintre, peut, dans des cas choisis, vivifier notre connaissance de réel, et surtout peut-être nous apprendre à réintroduire nous-même, avec combien de prudence, certes, de cette subjectivité dans nos évocations?

Les peintres suggèrent la durée intérieure et nous rappellent que tout homme regarde le cadre dans lequel il vit à travers des lentilles affectives: Amiel disait d'un paysage qu'il était un état d'âme. En plus de leurs sentiments particuliers, ils font passer dans leurs tableaux - probablement à leur corps défendant - l'esprit de leur époque: l'on en conviendra aisément. Ne livrent-ils pas, par là même, une composante supplémentaire du paysage? Car la manière qu'ont les hommes de regarder le milieu où ils vivent et qu'ils façonnent, ne doit-elle pas entrer aussi dans une appréciation du monde qui se veut complète? Pour tout dire, les peintres ne sont-ils pas détenteurs sinon d'un secret perdu, 
du moins d'un secret que n'arrive pas à retenir, pas à fixer bien souvent ni la photo, ni la description: celui d'une prise de conscience que la vérité d'un paysage doit être vécue autant qu'analysée?

De cette perception supplémentaire en somme, il est donné à bien des gens d'en éprouver la réalité. Comment expliquer autrement l'attrait renouvelé qu'exerce une exposition de tableaux?

Il existe bien de photos de bidonvilles sordides; mais la réalité de misère silencieuse et de bricolage ne fuse-t-elle pas plus subtilement dans l'esprit de celui qui regarde un morceau de «zone» peint par Laporte? La composition de Klee, «Devant les portes de Kairouan»: le jeu de coloris d'un non-conformisme des formes? Mais qui ne referait pas devant cette toile l'expérience proustienne de l'évocation d'une société à partir d'un détail retrouvé? La photo-vérité ne recrée pas cette présence qu'apporte un bon tableau. Par pudeur, par scrupule, le géographe refuse d'accorder droit de cité à cette troisième dimension émotionnelle, sans laquelle le monde se présente comme projeté à plat, extérieur à nous et non pas nous enveloppant. Quel livre pourra jamais nous réintroduire dans le complexe subtil de lac plat et de temps clair, et de vignes en foules ordonnées, spectatrices de la grande houle pétrifiée des Préalpes, autant qu'une «Vue de Chexbres» de Hodler? Où donc ailleurs qu'à travers l'un des brouillards jaunes de Marquet saisir dans leurs interférences, le bruit, l'activité et la morphologie du cœur de Paris?

L'autre fonction majeure du tableau est d'aider le géographe dans ce dialogue qu'il mène avec le visible. Pour appréhender le réel, la voie directe n'est pas nécessairement la plus efficace. Le relai de l'imaginaire - de la peinture en l'occurrence - si incomplet ou chargé d'erreurs qu'il soit, peut rendre service. L'art médiateur: l'idée n'est pas neuve. Mais elle n'a jamais eu d'écho dans notre discipline. Deux aspects de cette médiation paraissent pourtant faciles à admettre.

En premier lieu, un tableau fixe l'attention. Comment, à une époque où tant d'images excellentes nous sont offertes à profusion pour chaque région du globe, ne pas redouter la saturation mentale? Et comment une toile ne nous frapperait-elle pas d'autant plus, désormais, par son côté insolite d'œuvre artisanale, coûteuse en efforts, très éloignée de cette sensation d'achèvement que procure la photo en couleur? Autour d'un tableau jouant le rôle de mordant, s'accrochent d'autres images qui, autrement, s'en fussent allées à la dérive. Réhabilitons les peintres amateurs, et ceux que l'on qualifie de dominicaux. Sous cet angle précis d'une propédeutique de l'esprit, leurs services valent ceux des plus grands.

Ensuite et surtout la peinture de paysage nous permet d'ordonner des observations diffuses. A la manière d'une lentille, le tableau capte en nous, pour les faire converger en une image d'une intensité plus vive des notations latentes, parce que non concourrantes. Nathalie Sarraute, dans un texte récent, reprend à son compte d'écrivain cette phrase de Paul Klee «L'art ne restitue pas le visible, il rend visible». Les géographes seront-ils d'accord?

La «Forêt vierge» d'Henri Rousseau dissonne avec les vues connues de l'Amazonie ou du Congo. Mais elle les éclaire, comme elle prépare l'esprit à les mieux regarder, en nous initiant à l'oppression et à la singularité de ce milieu végétal. Et pourtant dans le détail, quoi de plus fantaisiste? Le «Manhattan» de Dufy, et celui de Buffet, ne le cèdent pas en extravagance, si l'on y regarde de près, à la forêt du Douanier. Mais à s'en laisser pénétrer, l'on s'enrichit de perceptions nouvelles: celle de la verticalité inhumaine des cités du Nouveau Monde ou du monde de demain, celle de l'activité foisonnante de la plus grande ville du globe. Aller de la Provence à Van Gogh, puis y revenir, voilà une dialectique dont beaucoup d'entre nous, plus ou moins consciemment, ont tiré profit. "Le paysage à l'arbre rose», de Gauguin aide celui qui l'accueille à «précipiter», à synthétiser ce qu'il a vu de la campagne bretonne. Son acuité d'observation s'en affermit pour de nouvelles investigations. Qui refuserait d'accorder à Pissarro, à Sisley, à Chapelain-Midy 
ces vertus médiatrices pour la connaissance de l'entre Loire et Seine, à Fougeron ou à Maeglin pour celle des paysages industriels?

Il va de soi que pour tous les cas où se noue ce dialogue à trois entre le géographe, le paysage et l'image qu'en donne l'artiste, il en est d'autres, infiniment plus nombreux, où faute de référence picturale, nous nous trouvons directement aux prises avec ce que nous devons décrire. Prendre conscience que, de ce fait, notre vision reste probablement en deçà de qu'elle pourrait être, ne constitue pas, je pense, le plus mince profit d'une telle recherche menée en marge des méthodes classiques.

\section{Tout n'a pas été peint}

L'insuffisance de la couverture de notre globe en tableaux de paysages demande l'analyse. Celle-ci seule, en effet, nous permettra de définir les limites concrètes de ce recours à la peinture.

Une première restriction nous vient souvent de l'angle de vue et du cadrage. Les peintres, qui paraissent ne jamais avoir ćté véritablement intéressés jusqu'à présent par la vue verticale du paysage (et l'on en demeure surpris, tant le manteau terrestre réserve d'inattendu et de variété à qui le regarde d'en haut) préfèrent, en règle générale, un champ visuel étroit et des motifs rapprochés. Sans doute les tableaux panoramiques ne manquent-ils pas, dans le genre de «L'espace» de Chaintreuil ou de «La vue de Montmartre» de Van Gogh. Des expériences même ont été tentées pour restituer l'immensité de l'horizon: au musée de Bâle, le panneau de Coghuf intitulé «Les quatre heures» mesure huit mètres de large sur quatre-vingt centimètres de haut. Mais la majorité des toiles appartiennent à la catégorie des gros plans ou des plans moyens.

Cette grande échelle de l'observation n'est pas étrangère au géographe, qui la pratique. Rien n'oblige en effet à embrasser de vastes panoramas pour saisír un phénomène dans son ensemble. Un élément choisi le suggère souvent d'une manière plus incisive. N'est-ce pas tout un type d'économie et d'habitat traditionnels des vallées du bassin parisien qui surgit à l'esprit devant «Les toits rouges» de Pissarro? «La petite rue»de Vermeer, si intime, si dépourvue de prétention, ne vaut-elle pas pour la ville et la vie hollandaise, et bien au delà du XVIIe siècle? Avec quelques pans de murs b!ancs et deux silhouettes ( Les Fellahs»), Van Dongen n'évoque-t-il pas des quartiers entiers de médina? Mais l'on regrette malgré tout que le large et le lointain n'aient pas davantage tenté les peintres. Leur centre d'intérêt se situe à un niveau analogue à celui du «type de temps». Principalement. Et subsidiairement au niveau du climat. Chez le géographe, l'échelle de préoccupation est inversée.

Une seconde limitation tient aux genres traités. Un relevé précis et un classement thématique des tableaux de paysages n'exis:e pas encore. Il vaudrait certainement la peine qu'on y prendrait. A défaut de ce document, l'on peut, sans grands risques d'erreurs, ranger parmi les favorisés, les sujets à dominante climatique et rurale. Le fait urbain n'est pas mal partagé non plus. La topographie en elle-même a peut-être moins inspiré. Encore qu'il y ait pour le morphologue, au total, une matière abondante: le relief ne se modifie guère avec les siècles, et telle œuvre ancienne détient facilement autant de puissance suggestive qu'une autre contemporaine. Le Quattrocento a dressé déjà une étonnante typologie calcaire. Mais les modernes - je pense à un Jongkind sur Grenoble, et à plusieurs Segantini traitant des Alpes - ont parfois des intuitions synthétiques: ils nous offrent des contrastes structuraux ou morphoclimatiques dignes de figurer dans un traité de géographie physique.

La grande défaillance, incontestablement, concerne les paysages industriels. A part quelques apôtres connus, les témoins, curieusement, manquent. Serait-ce parce que l'art, et donc la culture, ainsi que le veulent certains philosophes, s'inscrivent en faux contre l'actuelle civilisation technicienne, subtile négatrice de l'homme? L'explication, quoiqu'il en soit, ne comble pas la lacune. 
Même les genres les plus représentés n'épuisent pas la réalité. Toutes les régions d'un pays et tous les pays du monde n'ont pas été peints. Ici encore, un pointage cartographique des lieux ayant fait l'objet d'un tableau serait le bienvenu. Il montrerait certainement des continents pauvres, et d'autres riches. La zone tropicale et les pays musulmans figureraient parmi les démunis: parce qu'ils correspondent à des domaines économiquement faibles du globe, et que la peinture suppose toujours un mécènat privé ou d'Etat, et donc des disponibilités financières pour une activité «inutile»; mais tout autant parce que l'art de ces régions s'est orienté vers d'autres modes d'expression. Ce dernier motif vaut en tout cas pour les pays d'Islam, où la représentation figurative est, comme on le sait, interdite. Les paysagistes de ces terres restées à l'écart du mouvement pictural, quand il y en a, viennent généralement des pays colonisateurs: les Post, les Chassériau, les Gauguin ...

Au contraire, l'Asie orientale sino-japonaise, et, largement prépondérante, l'Europe (avec ses prolongements ethniques récents), accuseraient les plus fortes densités d'œuvres peintes. Les causes - immédiates au moins -, se devinent aisément: elles sont économiques, sociales et spirituelles comme dans les pays pauvres en peinture, mais pour des raisons opposées.

L'on noterait ensuite qu'à l'intérieur de cette Europe privilégiée, l'homogénéité ne règne pas. Le génie national intervient, et crée des différences. La France a engendré beaucoup plus de peintres que l'Allemagne.

Enfin, au cocur même de cette France qui inspira tant de paysagistes, surtout au moment de l'impressionnisme, le bassin parisien, la Normandie, la Bretagne et la Provence attirèrent seuls, ou presque seuls. Pourquoi plus de la moitié du pays fut-elle délaissée?

Chaque époque - et particulièrement celle que nous vivons, et qui, pour nous, présente le plus d'intérêt - n'a pas éprouvé une inclination équivalente pour la peinture de l'extérieur. A ne considérer évidemment que le seul paysage, et avec toute la prudence que se doit de manifester un profane en matière d'histoire de l'art, je distinguerais volontiers trois périodes majeures dans l'évolution de la peinture. Cette articulation correspond à des changements dans les courants de pensée et, notamment, dans le sentiment de la nature. Mais elle est liée aussi, plus ou moins directement, au développement de techniques telles que le cinéma ou la photo en couleur.

Au cours d'une première phase, antéromantique, et à l'exception combien importante, il est vrai, de nombreuses œuvres des écoles flamandes et hollandaises, le paysage, quand il ne cède pas au goût baroque, joue avant tout un rôle de cadre. Que l'on se souvienne de cette «Prise de Jéricho» dans les Heures d'Etienne Chevalier, où timide et exquis, en fond de scène, se tient tout un côteau de la Loire; que l'on songe encore à ces piémonts alpins qui se profilent, l'un délicat et scrupuleux derrière la «Vierge d'Autun» de Van Eyck, et l'autre brumeux et un peu fantastique à l'arrière-plan du célèbre trio de Vinci ( «La Vierge, l'Enfant Jésus et Sainte Anne»).

Une seconde période s'ouvre, à la fin du XVIIIe siècle, avec la découverte romantique de la nature. Elle durera une centaine d'années. Le paysage devient alors objet d'intérêt en soi. L'Angleterre et la France, entre autres, s'y passionnent.

A l'époque contemporaine, enfin, en dépit d'une phalange encore nombreuse de peintres figuratifs, il tend à ne plus être qu'un prétexte à spéculations esthétiques. Après les Turner, les Sisley et les Van Gogh, et malgré les Marquet et les Buffet, beaucoup de peintres s'acheminent vers l'informel. Un «Paysage du Midi» de Picasso devient cacophonie - incontestablement méditerranéenne et probablement marqué au coin du sceau du génie -; une «Vue de Caux» de Hodler frise la mystification; les compositions de Rouault appartiennent à un autre monde.

Or, si les tableaux anciens restent des documents technologiques, sociologiques, et pour tout dire paléographiques d'autant plus précieux qu'aucun témoignage photographique ne s'y substituera jamais, ils ne nous aident guère pour la compréhension du 
monde actuel, notre domaine. Il apparaît alors que pour ce qui est des dernières décennies nous avons joué de malchance. A l'âge d'or de la peinture de paysage, qui culmine avec l'impressionnisme, les sciences descriptives de la terre recrutaient peu: elles s'essayaient. Aujourd'hui que leur maturité ne fait plus de doute, et que des contacts transdisciplinaires nous profiteraient, l'art se dérobe. Dans une certaine mesure peintres et géographes se sont croisés.

Que reste-t-il donc, en dernière analyse, de cette contribution de la peinture à la géographie? La peinture apparaît bien comme prise en tenaille entre deux limites: d'une part, cette difficulté fondamentale qu'est son manque d'objectivité, et d'autre part, d'importantes lacunes de fait, puisque les peintres n'ont pas fixé tous les paysages, loin de là, sur leurs toiles.

Une attitude réservée semble donc s'imposer. Et pourtant ne perdrait-on pas quelque chose à s'en tenir à l'actuelle pauvreté d'échanges? Notre vision de géographe peut-elle rester une perception strictement «scientifique»? Ne doit-elle pas, sans pour autant abdiquer l'esprit critique, accepter l'idée d'une enquête plus large ayant éventuellement recours à l'art? Est-il vraiment paradoxal de penser qu'en acceptant ce dernier nous avons chance de gagner, dans les cas favorables, cette dimension émotionnelle supplémentaire qui confère au réel tout son volume, et qu'en outre, la référence à un monde imaginaire peut servir d'étape précieuse dans notre approche tâtonnante de ce qui est?

Une recherche en tout cas ouverte à cette direction serait-elle contraire à la vraie prudence?

\title{
MALEREI UND GEOGRAPHIE
}

Vermögen die Gemälde der Maler, insbesondere diejenigen der Landschaftsmaler, etwas Wesentliches zu unserer «wissenschaftlichen»Vision der Ende beizutragen? Sind sie imstande, uns Aspekte der Realität zu enthüllen, die wir selbst keineswegs immer zu sehen verstehen? In verschiedener Hinsicht scheint diese Frage paradox zu sein. Auf der einen Seite sind die Auffassungen des Künstlers zweifellos von den unsrigen verschieden, und seine Sprache ist keineswegs objektiv. Andrerseits verfügen auch wir aus verschiedenen Gründen nur über ein sehr unvollständiges «Oberflächenbild» der Landschaften. Just daher bleibt es nicht weniger wahr, daß die Kunst des Malers, insbesondere da, wo sie sich von unseren wissenschaftlichen Verfahren unterscheidet, uns zunächst lehrt, ein Gemütsmaß in das Verständnis der Erde einzufügen, die wir zu beschreiben trachten, sodann aber uns in dialektischer Weise hilft, deren Realität zureichender zu begreifen.

Diese Berufung auf die Bildkunst erscheint deshalb letzten Endes, in einem weitern Rahmen der geographischen Erkenntnis und wohlverstanden sehr vorsichtig angewendet, als ausgesprochend bereichernd.

\section{PROBLEME DER QUARTÄRFORSCHUNG IN EUROPA}

\author{
Ein Tagungsbericht 1 \\ DIETRICH BARSCH
}

Vom 8.-12. Oktober 1964 fand in Lüneburg/Niedersachsen die 12. Tagung der Deuqua (Deutsche Quartärvereinigung) statt. Sie vereinigte etwa 130 am Quartär interessierte Geographen, Geologen, Pedologen, Botaniker, Zoologen und Prähistoriker aus Deutschland, der Schweiz, Belgien und den Niederlanden. Neben allgemeinen Problemen der Eiszeitforschung wurden vor allem - dem genius loci entsprechend -

1 Die Teilnahme an der hochinteressanten und anregenden Tagung der Deuqua in Lüneburg war mir aufgrund eines großen Entgegenkommens von Herrn Prof. Dr. H. Annaheim möglich, dem ich auch an dieser Stelle dafür meinen herzlichen Dank aussprechen möchte. 